Check for updates

Cite this: RSC Adv., 2019, 9, 17148

Received 24th April 2019

Accepted 8th May 2019

DOI: 10.1039/c9ra03064f

rsc.li/rsc-advances

\section{Rationally designed La and Se co-doped bismuth ferrites with controlled bandgap for visible light photocatalysis}

\author{
M. Umar, ${ }^{a}$ Nasir Mahmood, (D) ${ }^{\mathrm{b}}$ Saif Ullah Awan, ${ }^{\mathrm{c}}$ Sabeen Fatima, ${ }^{\mathrm{a}}$ Asif Mahmood ${ }^{\star d}$ \\ and Syed Rizwan (iD *a
}

Development of efficient visible light photocatalysts for water purification and hydrogen production by water splitting has been quite challenging. The activities of visible light photocatalysts are generally controlled by the extent of absorption of incident light, band gap, exposure of catalyst surface to incident light and adsorbing species. Here, we have synthesized nanostructured, La and Se co-doped bismuth ferrite (BLFSO) nanosheets using double solvent sol-gel and co-precipitation methods. Structural analysis revealed that the $\mathrm{La}$ and $\mathrm{Se}$ co-doped BFO i.e. $\mathrm{Bi}_{0.92} \mathrm{La}_{0.08} \mathrm{Fe}_{1-x} \mathrm{Se}_{x} \mathrm{O}_{3}$ (BLFSO) transformed from perovskite rhombohedral to orthorhombic phase. As a result of co-doping and phase transition, a significant decrease in the band gap from $2.04 \mathrm{eV}$ to $1.76 \mathrm{eV}$ was observed for BLFSO-50\% (having Se doping of 50\%) which requires less energy during transfer of electrons from the valence to the conduction band and ultimately enhances the photocatalytic activity. Moreover, upon increase in Se doping, the BLFSO morphology gradually changed from particles to nanosheets. Among various products, BLFSO-50\% exhibited the highest photocatalytic activities under visible light owing to homogenous phase distribution, regular sheet type morphology and larger contact with dye containing solutions. In summary, La, Se co-doping is an effective approach to tune the electronic structure of photocatalysts for visible light photocatalysis.

\section{Introduction}

Nanoscale semiconductor-based photocatalysts have elicited significant consideration over the last decades due to their possible applications in solar energy transformation and environmental purification. ${ }^{1}$ Amongst them, titanium dioxide $\left(\mathrm{TiO}_{2}\right)$ has been demonstrated to be a dominant photocatalyst for the degradation of abundant organic amalgams ${ }^{2}$ despite the fact that it can only react to UV light due to its wide bandgap $(\sim 3.2 \mathrm{eV})$. It is well known that the morphology, bandstructures, and nanocomposites with various rare-earth metals could be the reasons for the improving photocatalytic activity of $\mathrm{TiO}_{2} \cdot{ }^{3-8}$

As solar energy comprises $47 \%$ visible and only $2 \%$ UV radiation, these photocatalysts have inadequate activity under

\footnotetext{
${ }^{a}$ Physics Characterization and Simulations Lab (PCSL), School of Natural Sciences (SNS), National University of Science and Technology (NUST), Islamabad 44000, Pakistan.E-mail: syedrizwanh83@gmail.com; Tel: +92 5190855599

${ }^{b}$ School of Electrical and Computer Engineering, RMIT University, 124 La Trobe Street, 3001 Melbourne, Victoria, Australia

${ }^{c}$ Department of Electrical Engineering, NUST College of Electrical and Mechanical Engineering, National University of Sciences and Technology (NUST), Islamabad 44000, Pakistan

${ }^{d}$ School of Chemical and Biomolecular Engineering, The University of Sydney, 2006, Sydney, Australia. E-mail: asif.mahmood@sydney.edu.au; Tel: +61 399255439
}

solar electromagnetic radiation. ${ }^{\mathbf{1 1}}$ To efficiently make use of solar energy that contains mostly visible light, it is critical to discover visible-light-driven photocatalysts. ${ }^{9}$ However, it is quite challenging to design visible light driven photocatalysts because of the large bandgap which limits excitation in the visible region and hence limits the catalytic performance of the designed nanomaterials. Several efforts have been made to design effective visible light driven photocatalysts, however, realization of photocatalysis in visible region is still far from reality.

In recent years, the bismuth ferrite $\mathrm{BiFeO}_{3}$ (BFO) has attracted great interest due to its multiferroics properties as this system shows the ferroelectricity and anti-ferromagnetism. ${ }^{\mathbf{1 0}}$ Besides its multiferroics property, current studies also revealed promising visible-light driven photocatalytic properties for BFO. ${ }^{12,13}$ The BFO as photocatalyst has attained a great attention due to its small band gap (2.2 to $2.7 \mathrm{eV}$ ), chemical stability, low cost and good response to visible-light irradiation. ${ }^{\mathbf{1 4}}$ Furthermore, magnetic properties of $\mathrm{BiFeO}_{3}$ can assist in reprocessing the photocatalyst after the reaction via external magnetic field. ${ }^{\mathbf{1 4}}$ This avoids the damage of the catalyst over the rotations and makes the catalyst cost-effective. There by, $\mathrm{BiFeO}_{3}$ can act as a noble candidate for visible-light photocatalytic degradation of organic pollutants/dyes. ${ }^{15}$ Still, as talented as BFO material is, a few shortcomings need to overcome prior to tangible 
commercial and industrial applications. One serious difficulty is the high leakage current in BFO materials, essentially initiating from numerous impurity phases e.g. $\mathrm{Bi}_{2} \mathrm{Fe}_{4} \mathrm{O}_{9}, \mathrm{Bi}_{25} \mathrm{FeO}_{40}$ and $\mathrm{Bi}_{36} \mathrm{Fe}_{24} \mathrm{O}_{57}$ as well as oxygen vacancies, resulting in poor photocatalytic behavior. ${ }^{16}$ In addition, relatively larger bandgap (2.2-2.7) further limits the excitation upon light irradiation. Owing to these shortcomings, the BFO based materials have exhibited very poor photocatalytic degradation efficiencies $\mathbf{1 4}^{\mathbf{1 4 - 2 1}}$ and the work is still going on in this field for improving the catalytic activity of multi ferric systems under visible light. Hence, it is pertinent to find ways to tailor the chemistry of BFO materials so as to obtain pure phase product with lower bandgap in order to harness maximum activities under visible light irradiation. ${ }^{22-26}$

Here, we present a straightforward methodology for tailoring the inherent structure of BFO in such a way that a single phase product could be obtained with much lower bandgap of $\sim 1.76 \mathrm{eV}$ having sheet type morphology for enhanced contact with incident light and adsorbing species. The product was synthesized by simple wet-chemical and double solvent sol-gel method. Keeping in mind the high probability of recombination between photo-generated electrons and holes in single phase products, we introduced secondary dopants in the form of lanthanum (La) and selenium (Se) to control the recombination and enhance the number of delocalized electrons. A series of experiments were carried out in order to understand the effect of dopant upon morphology, and bandgap and the resulting photocatalytic activity for co-doped (La, Se) BFO nanosheets. Structural changes, morphologies, electronic properties and optical measurement were performed to analyze the recombination rate of charge carriers and elemental composition of the co-doped series. To the best of our knowledge, this is the first study which reports the control of inherent BFO structure via La and Se.

\section{Experimental details}

A series of nanoparticles of (La, Se) co-doped BFO with empirical formula $\mathrm{Bi}_{0.92} \mathrm{La}_{0.08} \mathrm{Fe}_{1-x} \mathrm{Se}_{x} \mathrm{O}_{3}, x=0,10 \%, 25 \%, 50 \%$, $100 \%$ labelled as BFO, BLFO, BLFSO-10\%, BLFSO-25\%, BLFSO$50 \%$, BLFSO-100\% were synthesized by double solvent sol-gel method. These samples are abbreviated as "BLFSO" with varying concentration of Se from $10 \%$ to $100 \%$, at fixed $8 \% \mathrm{La}$ concentration. All chemicals were purchased from Sigma Aldrich commercial grade and used without further modification. The precursors of $\mathrm{Bi}\left(\mathrm{NO}_{3}\right)_{3} \cdot 5 \mathrm{H}_{2} \mathrm{O}$ (99.90\%, pure) and $\mathrm{La}\left(\mathrm{NO}_{3}\right)_{3} \cdot 6 \mathrm{H}_{2} \mathrm{O}(99.90 \%$, pure) were stoichiometrically grinded and both were dissolved in ethylene glycol $\left(\mathrm{C}_{2} \mathrm{H}_{6} \mathrm{O}_{2}\right)$ and acetic acid $\left(\mathrm{C}_{2} \mathrm{H}_{4} \mathrm{O}_{2}\right)$ with continuing stirring for $2 \mathrm{~h}$ at room temperature. On the other hand, $\mathrm{Fe}\left(\mathrm{NO}_{3}\right)_{3} \cdot 9 \mathrm{H}_{2} \mathrm{O}(99.50 \%$, pure $)$ and Se powder (99.50\% pure) were dissolved in acetic acid and stirred for $2 \mathrm{~h}$. Both the prepared solutions were again mixed and stirred for $3 \mathrm{~h}$ on magnetic stirring. After that, the solution was dried in an oven at $90{ }^{\circ} \mathrm{C}$ for $12 \mathrm{~h}$ to get a gel and then calcined in furnace at $600{ }^{\circ} \mathrm{C}$ for $3 \mathrm{~h}$. For pure BFO, similar preparation methods have been demonstrated earlier. ${ }^{26}$ Structural analysis of the all samples were performed by X-ray
Diffractometer (XRD) in the range of $2 \theta=20-80^{\circ}$ using $\mathrm{Cu}-\mathrm{K} \alpha$ $(\lambda=0.15418 \mathrm{~nm})$ radiation with a scanning speed of 2 degree per minute. Field emission electron microscopy (FESEM, Hitachi-S5500) was used for study of the microstructures and morphological analysis. The photon source was an Al K $\alpha(1486.6$ eV) source operating at $350 \mathrm{~W}$. He-Cd laser of wavelength $325 \mathrm{~nm}$ was used as the excitation source during room temperature photoluminescence (PL) measurement. The PL spectrum was observed using a Hitachi luminescence spectrometer (F-4500). Room temperature UV-Vis absorption spectroscopy measurements were carried out using PerkinElmer, Lambda 950 photo spectrometer system.

\section{Results analysis and discussion}

The XRD analysis was used to investigate the crystalline structure of BFO and derived nanomaterials and the results are presented in Fig. 1. The XRD patterns of pure BFO (JCPDS card no. 20-0169 and no. 42-0201) corresponds to rhombohedral structure with distorted perovskite structure corresponding to $R 3 c$ space group. ${ }^{27-29}$ The dopants were systematically introduced into the BFO crystal lattice. The La was firstly introduced into the crystal lattice and with about $8 \%$ addition of $\mathrm{La}$, a phase pure BLFO having a composition of $\mathrm{Bi}_{0.92} \mathrm{La}_{0.08} \mathrm{FeO}_{3}$ was obtained as revealed by XRD analysis (Fig. 1). After fixing the La doping concentration to $8 \%$, the secondary dopant was introduced into the La doped BFO. Remarkably, (012) peak of pure BFO sample disappeared on increase in the Se concentration from $x=0 \%$ to $x=100 \%$ and structural phase transformation from rhombohedral to orthorhombic crystal structure was observed. In addition, a slight shift in the peak positions corresponding to the (104) and (110) towards higher angles were observed with Se co-doping in BLFO nanoparticles which could be ascribed to difference in ionic radius of dopants $(\mathrm{Se}=0.64 \AA$, $\mathrm{La}=1.032 \AA)$ than parent ions $\left(\mathrm{Bi}^{3+}=1.03 \AA\right)$, Fe $\left(\mathrm{Fe}^{3+}=0.78 \AA\right) .{ }^{30-34}$ The XRD analysis revealed that the Se codoping plays a critical role in phase transformation of pure BFO from rhombohedral to orthorhombic phase. The compositions with relatively lower doping contents $(x=0 \%, 10 \%$ and $25 \%)$ exhibited mixed phases i.e. rhombohedral and orthorhombic. In contrast, compositions with relatively higher doping ( $x=50 \%$ and 100\%) exhibited pure orthorhombic phase.

To further look into the impact of co-dopant on the morphology of BFO, scanning electron microscope (SEM) measurement were carried out and presented in Fig. 2(a-e). An overall particle type morphology was observed for BLFO as depicted in Fig. 2(a). The doping of Se lead to significant change in particle type morphology of BLFO and resulting Se-doped products exhibit an overall sheet-type morphology. Relatively thin sheets were observed with lower doping of Se $(10 \%)$ as presented in Fig. 2(b). Subsequent increase in Se concentration lead to relatively larger sheets possibly due to long range 2dimensional order in the structure as shown in Fig. 2(c). The product obtained with Se doping of $\sim 50 \%$ (Fig. 2 (d)) exhibited large and compact sheets which ensure exposure of large surfaces for enhanced catalytic response. Going beyond 50\% Se doping caused distortions in the sheets leading to irregular 

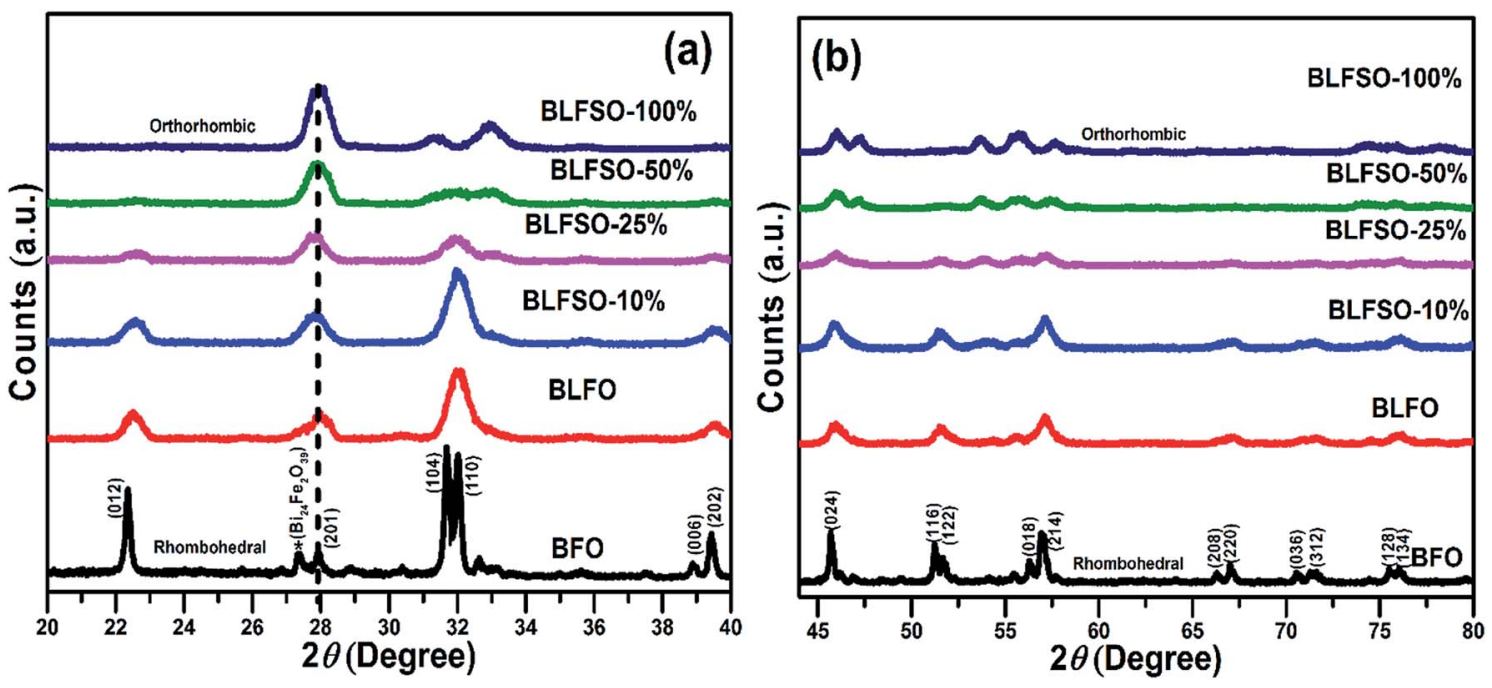

Fig. 1 The XRD pattern of BLFSO with varying concentration of Se (a) 20-40 and (b) 42-80 degrees.

structure and hence compromised the overall surface of the product as shown in Fig. 2(e).

Room-temperature photoluminescence (PL) emissions at the excitation wavelength of $300 \mathrm{~nm}$ were measured to investigate the effect of co-dopants for generation of possible defects within the narrow bandgap of pure BFO system. Modification of band structure in a photocatalyst is fundamentally related to interruption or reduction in the recombination opportunities through the photocatalytic progression. ${ }^{34-40}$ Fig. 3 shows the PL spectra of pure and co-doped BFO samples. The PL spectra of each sample has asymmetric broad curve with clear peaks identifications at $357 \mathrm{~nm}, 378 \mathrm{~nm}$, and $400 \mathrm{~nm}$. The characteristic PL signal at $357 \mathrm{~nm}$ is ascribed to the radiative emission of nanostructures during the recombination of electron-hole pairs, while the other signals at $378 \mathrm{~nm}, 400 \mathrm{~nm}$ are due to the emission as a result of the recombination owing to the defects, and donor-acceptor interactions. ${ }^{41-44} \mathrm{~A}$ small kink corresponding $455 \mathrm{~nm}$ was observed in pure $\mathrm{BFO}$ which correspond to bandgap of $2.72 \mathrm{eV}$. In comparison to BFO, the BLFSO-50\% exhibited a much shorter bandgap of $2.43 \mathrm{eV}$ at emission wavelength of $510 \mathrm{~nm}$. Interestingly, no impurity was observed close to $520 \mathrm{~nm}$ in BFO system as reported previously. However, we noticed an impurity peak only existing in pure BFO nanoparticles at $623 \mathrm{~nm}$. Additionally, broad spectra in the range of 640-700 nm (inset Fig. 3) was observed for BLFSO-50\% and BLFSO-100\% sample. No such peak at higher wavelength was observed for BFO. It can be speculated that the intense spectrum arise due to the phase transition from rhombohedral to orthorhombic occurred upon increasing Se concentration in the derived products.

Photocatalytic activity is strongly affected by competition between the charge separation and recombination processes and the PL emission spectra have been widely used to estimate the rate of charge recombination. ${ }^{45,46}$ According to the previous studies, the lower the PL emission intensity, the lower is the recombination rate of the photo-generated electron-hole pairs and the greater is the photo-activity of photo-catalyst. ${ }^{47}$ It should be noted that no wavelength shift was observed upon doping of Se in the BFO, rather a strong variation in PL intensity was observed which clearly indicates the recombination behavior in the developed products. For instance, highest intensity of the major signal at $378 \mathrm{~nm}$ was observed for BFO which was greatly reduced for co-doped nanostructures particularly for BLFSO-50\%. Lower PL intensity for BLFSO-50\% nanosheets suggested reduction of radiative emission due to the improved recombination resistance owing to the de-localization of excited electrons in the conduction band. This indicates the possibility of enhanced photocatalytic response, higher stability and durability for the BLFSO-50\% nanosheets. In comparison to BLFSO-50\%, the PL analysis for BLFO- $100 \%$ exhibited higher radiative emissions clearly indicating higher recombination rate for higher doping of Se leading to poor catalytic response. It can be assumed that the Se doping of $50 \%$ would provide optimal ratio between $\mathrm{La}^{3+}$ and $\mathrm{Se}^{4+}$ dopants to tailor the intrinsic structure, hence leading to enhanced recombination resistance.

$\mathrm{X}$-ray photoelectron spectrum has been performed for evaluating the surface chemical states of the BLFSO sample. The peaks corresponding to Bi4f, Bi4d, C1s, O1s, La3d and Se 3d can be shown in Fig. 4. Selenium has two main peaks at $55.4 \mathrm{eV}$ and $59.1 \mathrm{eV}$ corresponding to the oxidation of BFO surface with selenium. Similarly, lanthanum has $834 \mathrm{eV}, 838.2 \mathrm{eV}, 851.3 \mathrm{eV}$ and $854.9 \mathrm{eV}$ corresponding to La3d $3 / 2$ and La3d5/2. Further C1s and O1s peaks are present at $298.8 \mathrm{eV}$ and $545.3 \mathrm{eV}$. One dominant peak corresponding to Bi4f and two peaks corresponding to Bi4d are clearly visible at binding energy of $168.8 \mathrm{eV}, 441 \mathrm{eV}$ and $466 \mathrm{eV}$. The presence of selenium and lanthanum peaks with a positive shift in the oxygen peak exhibits the bonding of La and Se over the BFO surface which in turns helps in enhancing the charge transfer over the BLFSO surface. ${ }^{48,49}$ The enhancement in charge transfer further reduces the recombination rate in the photocatalytic materials which further helps the photo catalyst in enhancing the dye degradation..$^{50}$ 

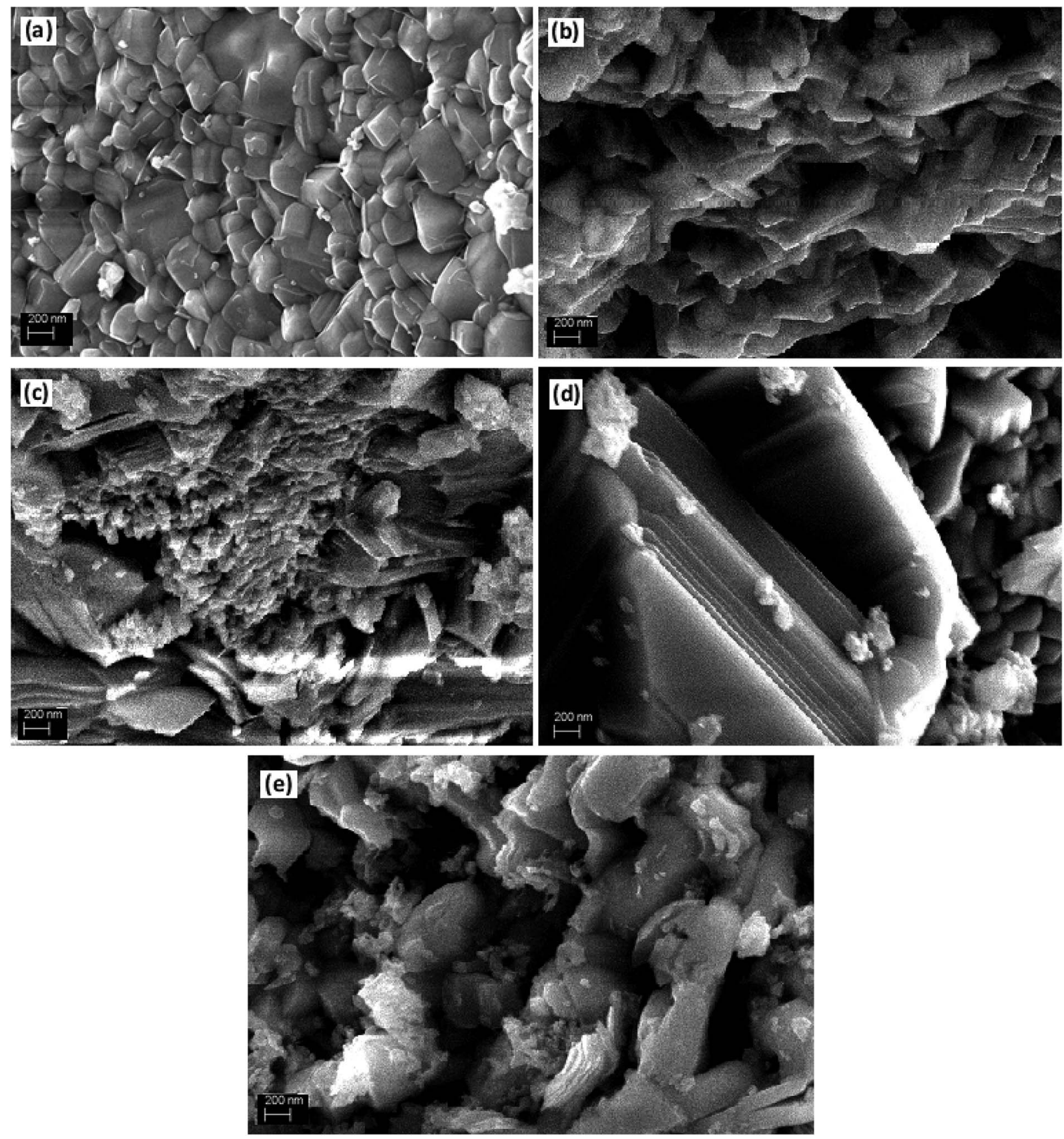

Fig. 2 SEM images of (a) BLFO, (b) BLFSO-10\% (c) BLFSO-25\% (d) BLFSO-50\% (e) BLFSO-100\%.

To investigate the influence of $\mathrm{La}^{3+}$ and $\mathrm{Se}^{4+}$ co-doping on the optical absorption of BFO, the UV-Vis diffused reflectance spectra (DRS) of the pure BFO and BLFSO samples were measured at room temperature, as shown in Fig. 5. The absorption band edge of the pure BFO nanoparticles appears at $610 \mathrm{~nm}$ which is comparable to previous reports ${ }^{51}$ and designates that BFO can react to visible light. In association with pure $\mathrm{BFO}$, the $\mathrm{La}^{3+}$ and $\mathrm{Se}^{4+}$ co-doped BFO samples displayed an enhanced absorption energy under visible light, and the absorption edge spectra change abruptly to the near infra-red region, which is a pure red shift change in these samples. Thus, the light absorption range is prolonged, which enhances the photocatalytic activity of BLFSO under UV-vis-NIR light treatment. Furthermore, the band-gaps were deliberated using the Kubelka-Munk function ${ }^{52}(\alpha h \nu)^{2}$ versus photon energy $(h \nu)$ for the direct band-gap semiconductor; the extrapolation of the tail at $y$-axis equals to zero gives the band gap, as shown in the inset of Fig. 5 The band-gaps are 2.03, 1.99, $1.96,1.80,1.77,2.05 \mathrm{eV}$ for the pure BFO, BLFO, BLFSO-10, BLFSO-25, BLFSO-50 and BLFSO-100 samples, respectively. The band-gap of the BLFSO-50 sample is smallest in comparison to that of BFO and other samples. The reduction in bandgap for BLFSO-50 can be ascribed to relatively larger crystals, optimally tailored lattice parameters and defects which all contribute to lowering of the band gap upon introduction of co-dopants. 


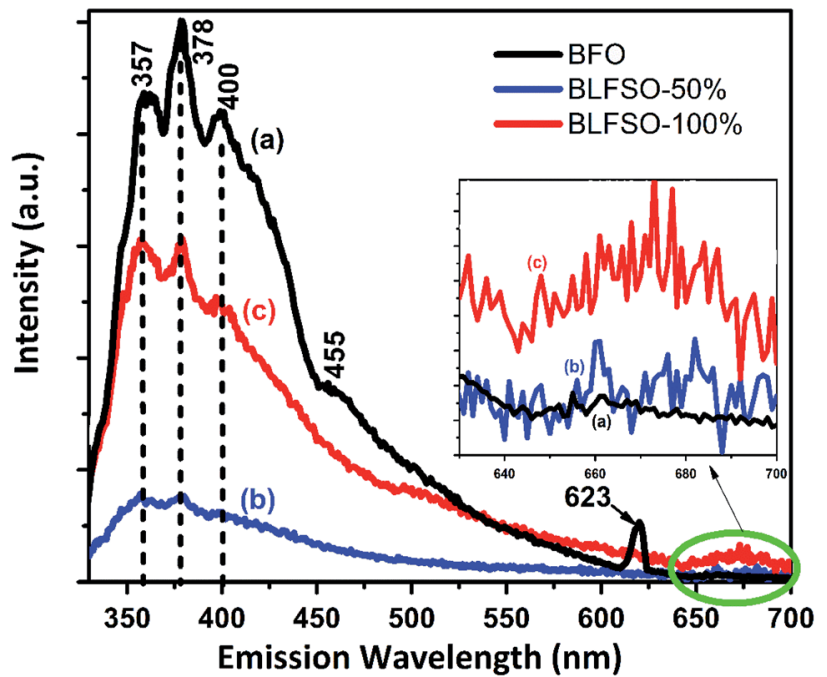

Fig. 3 Room temperature PL spectra of nanostructured (a) BFO (b) BLFSO-50\% (c) BLFSO-100\%.

The photocatalytic activity of the developed samples was investigated under visible light irradiation using xenon lamp having $300 \mathrm{~W}$ power source with $800 \mathrm{~nm}$ filters. The Congo Red (CR) was used was used as a model dye in aqueous solution. Fig. 6 represents the photocatalytic activity for various samples as a function of time. The dye solution exhibited limited dye degradation under visible light when no catalyst was used. However, upon addition of designed catalyst, much faster dye degradation was observed probably due to enhanced catalytic reaction on the surface of the catalyst. Highest levels of dye degradation were observed for BLFSO-50\% with over $90 \%$ dye degradation in first 30 minutes. The degradation level of the BLFSO nanoparticles is higher than the La doped BFO nanofibers ${ }^{51}$ and Se doped BFO. ${ }^{26}$ The dye removal time taken by the BLFSO-50\% is very less ass compared to other multi ferric nanoparticle systems (180 $\mathrm{min}, 270 \mathrm{~min}, 1 \mathrm{~h}$ and)..$^{\mathbf{4 0 5 2 - 5 4}} \mathrm{In}$ comparison, compositions with other Se doping levels exhibited much poor dye degradation activities. Higher dye degradation activities for BLFSO-50\% can be attributed to complete phase transition from rhombohedral to orthorhombic which provide favorable bandgap $(\sim 1.77 \mathrm{eV})$ and binding energies for enhanced catalysis of dye species. Moreover, homogenous and compact sheet type morphology provide higher active surface area and larger contact with dye solution which eventually enhance the number of exposed sites for catalytic reaction. The activities of compositions with lower Se doping i.e. BLFSO-10\% and BLFSO-25\% were limited by incomplete phase transition which limits the photocatalytic activity. Similarly, the BLFSO$100 \%$ exhibited much lower photocatalytic activities (58\%) which might be attributed to disruption of sheet type morphology which limits the contact with dye containing solution. Moreover, the stability of a photocatalyst is an important factor for its practical applicability. So, the BLFSO$50 \%$ photocatalyst were analyzed for four cyclic runs and as expected it showed approximately the same activity after repeating the photocatalytic process under visible light which confirmed its stability as shown in the Fig. 7.

The mechanism of photocatalytic reaction was investigated to obtain more insights into higher activities obtained from BLFSO samples. Generally, the photocatalysis usually involves three steps: (i) absorption of photons (ii) the production, separation of photo generated $\mathrm{e}^{-}-\mathrm{h}^{+}$pair (iii) redox reactions. In this process, $\cdot \mathrm{OH}$ radicals act as active specie for degradation of organic compounds such as the CR. ${ }^{55-61}$ These radicals are produced by capturing of their $\mathrm{e}^{-}-$ $\mathrm{h}^{+}$pair by molecular oxygen and water as described in the following equations:
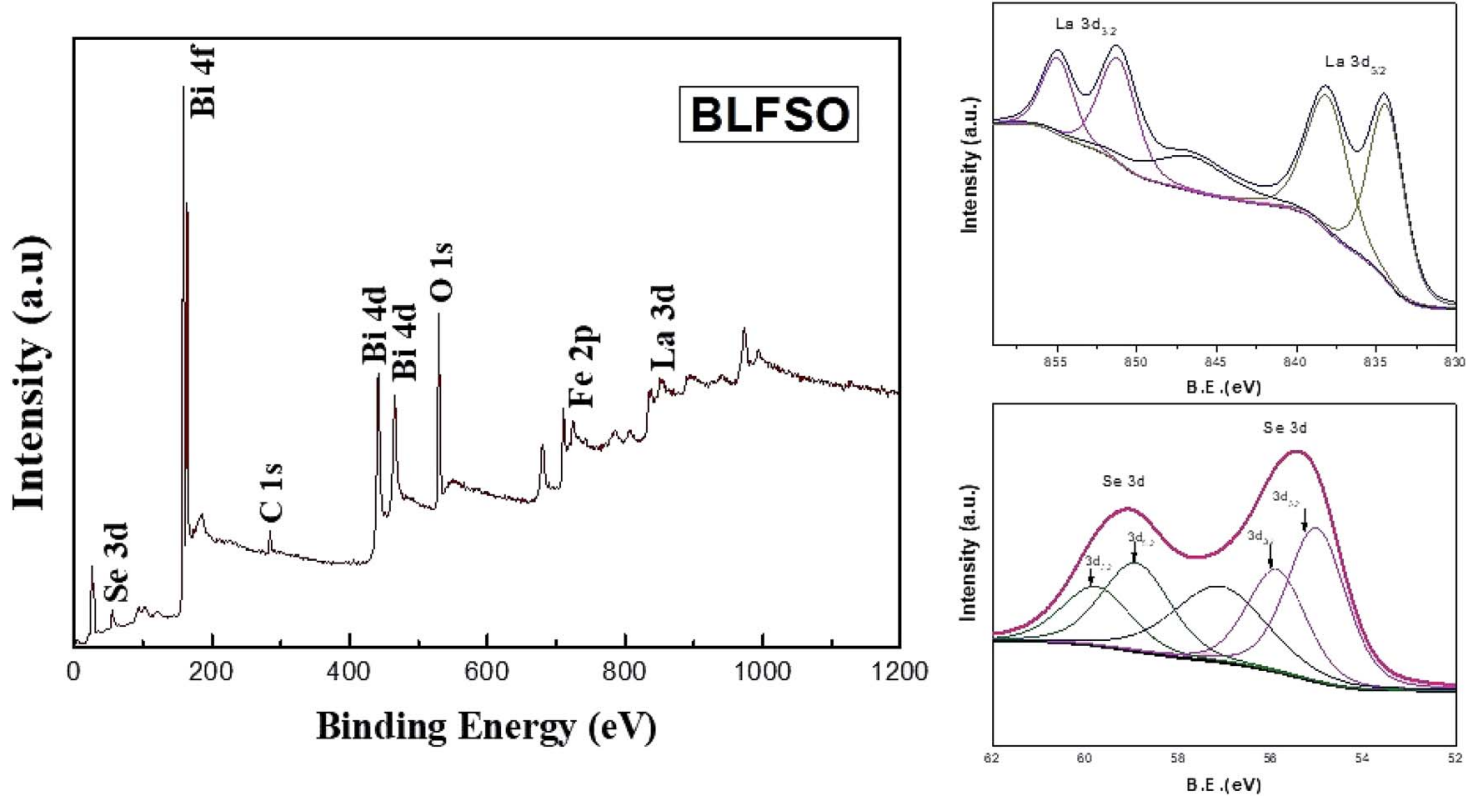

Fig. 4 XPS spectra of BLFSO sample. 


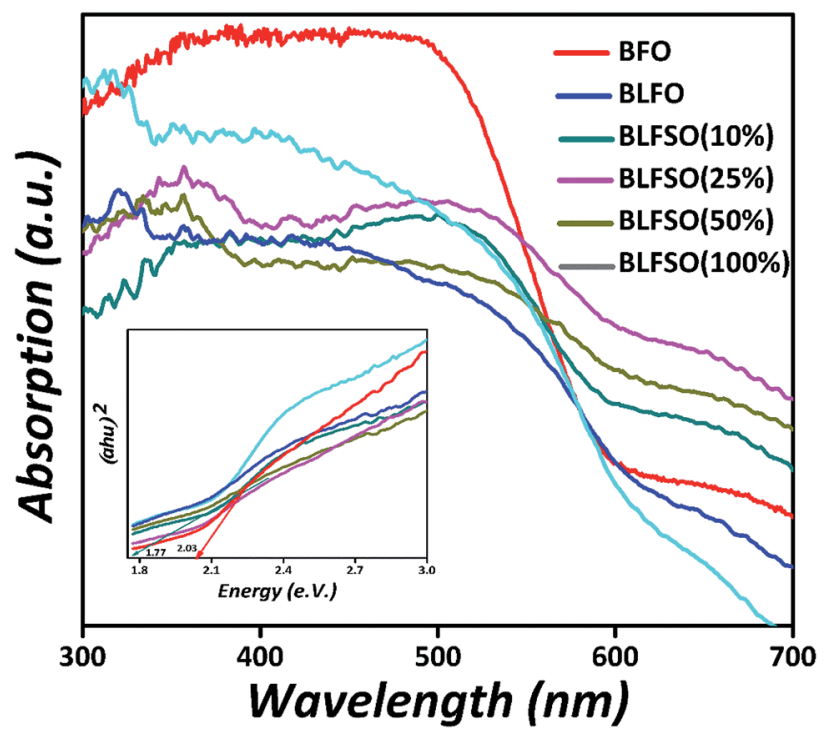

Fig. 5 Optical absorption spectra of BLFSO samples.

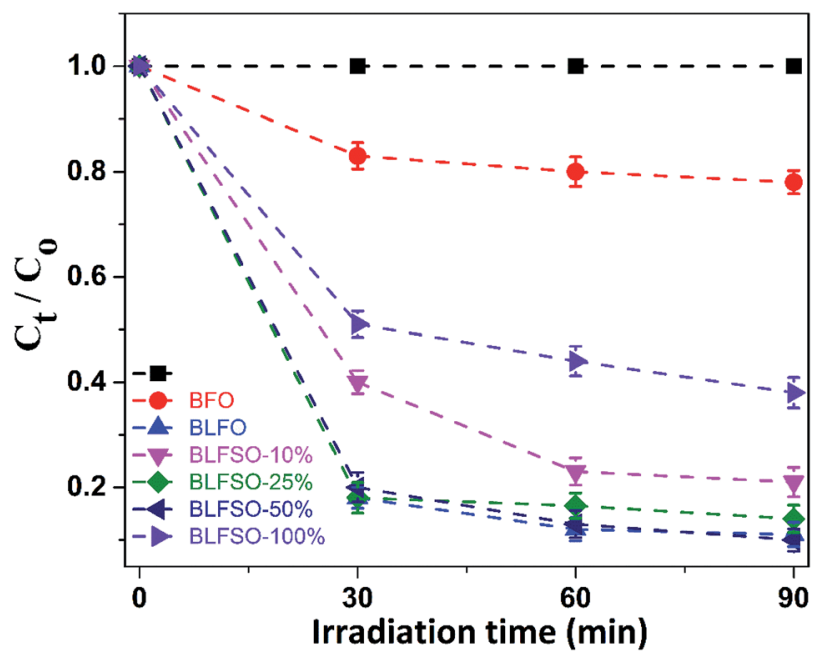

Fig. 6 The photo-degradation efficiencies of CR as a function of irradiation time under visible-light for BLFSO series of samples with varying the Se concentration from 10-100\%.

$$
\begin{gathered}
\text { Sample }+h \nu \rightarrow \text { sample* }\left(\mathrm{e}_{\mathrm{CB}}{ }^{-}+\mathrm{h}_{\mathrm{VB}}{ }^{+}\right) \\
\mathrm{h}_{\mathrm{VB}^{+}}+\mathrm{CR} \rightarrow \text { direct oxidated dye } \\
\mathrm{h}_{\mathrm{VB}^{+}}+\mathrm{H}_{2} \mathrm{O} \rightarrow \cdot \mathrm{OH}+\mathrm{H}^{+} \\
\mathrm{h}_{\mathrm{VB}^{+}}+\mathrm{OH}^{-} \rightarrow \cdot \mathrm{OH} \\
\mathrm{e}_{\mathrm{CB}_{-}}+\mathrm{O}_{2} \rightarrow \cdot \mathrm{O}_{2}^{-} \\
\cdot \mathrm{OH}+\mathrm{CR} \rightarrow \mathrm{CO}_{2}+\mathrm{H}_{2} \mathrm{O} \text { (by-products) } \\
\cdot \mathrm{O}_{2}^{-}+\mathrm{CR} \rightarrow \mathrm{CO}_{2}+\mathrm{H}_{2} \mathrm{O} \text { (by-products) }
\end{gathered}
$$

These radicals react with organic pollutant (CR in our case) and degrades it into harmless by-products (eqn (VI) and (VII)). ${ }^{56,62}$

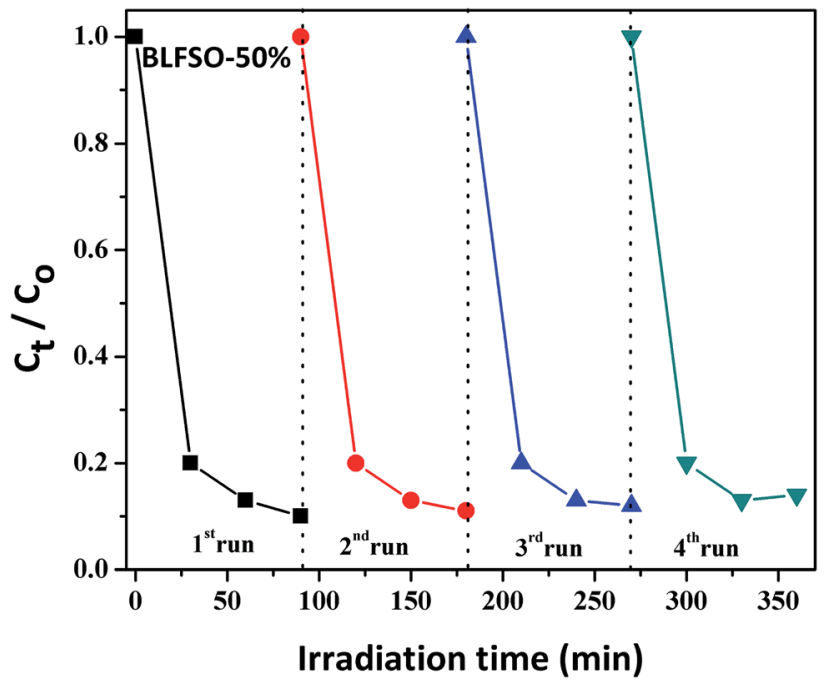

Fig. 7 The stability of BLFSO-50\% photocatalyst after four cyclic runs under visible light.

\section{Conclusion}

In summary, we report the tailoring of BFO structure with rational addition of dopants to control the inherent crystal structure and obtain much lower bandgap for enhanced visible light photocatalytic response. A series of samples with fixed La concentration and varying concentration of Se $(10 \%, 25 \%, 50 \%$, $100 \%$ ) were synthesized via simple double solvent sol-gel method and co-precipitation methods. The experimentation revealed that the bandgap of the co-doped samples could be easily tailored by varying the dopant contents and an overall sheet type product could be obtained. The lower bandgap provided easy electron availability upon exposure to incident radiation while the sheet type morphology ensured larger contact between surface of the catalyst and the adsorbing species, thus resulting in enhanced synergistic response and higher catalytic activities. Owing to these attributes, the developed product exhibited excellent photocatalytic activities for model dye, catalyzing more than $90 \%$ dye in first 30 minutes of exposure to visible light. We strongly believe that the methodology provide pathway for tailoring inherent structure of ferritebased catalysts to be used for visible light photocatalysis.

\section{Author's contribution}

M. Umar fabricated and characterized the samples, Saif Ullah Awan contributed in extensive manuscript writing and analysis of results, Nasir Mahmood, helped in characterization, Sabeen Fatima provided help with the XPS measurement and analysis of the sample. Asif Mahmood and Syed Rizwan conceived the research idea and co-supervised/supervised the entire project.

\section{Conflicts of interest}

There are no conflicts to declare. 


\section{Acknowledgements}

The authors are thankful to Higher Education Commission (HEC) of Pakistan for providing research funding under the Project No: 6040/Federal/NRPU/R\&D/HEC/2016 and HEC/ USAID for financial support under the Project No: HEC/R\&D/ PAK-US/2017/783. The author also thanks School of Natural Sciences (SNS) at National University of Science \& Technology (NUST), Islamabad, Pakistan, RMIT University, Australia and University of Sydney for technical and financial support.

\section{References}

1 Y. Ma, X. Wang, Y. Jia, X. Chen, H. Han and C. Li, Titanium dioxide-based nanomaterials for photocatalytic fuel generations, Chem. Rev., 2014, 114, 9987-10043.

2 D. Kanakaraju, B. D. Glass and M. Oelgemöller, Titanium dioxide photocatalysis for pharmaceutical wastewater treatment, Environ. Chem. Lett., 2014, 12, 27-47.

3 S. C. Yang, G. Chang, G. J. Yang, Y. J. Wang and B. Fang, Photocatalytic hydrogen generation from water reduction using orchestrated photosensitizers, Catal. Sci. Technol, 2015, 5, 228-233.

4 D. Xu, Q. Q. Chu, Z. Wu, Q. Chen and B. Fang, J. Catal., 2015, 325, 118-127.

5 Y.-J. Wang, G. Chang, Q. Chen, G.-J. Yang, S.-Q. Fan and B. Fang, Orchestrated photocatalytic hydrogen generation using surface-adsorbing iridium photosensitizers, Chem. Commun., 2015, 51, 685-688.

6 G. Liao, J. Fang, Q. Li, S. Li, Z. Xu and B. Fang, Ag-Based nanocomposites: synthesis and applications in catalysis, Nanoscale, 2019, 11, 7062-7096.

7 G. Liao, Y. Gong, L. Zhang, H. Gao, G.-J. Yang and B. Fang, Semiconductor polymeric graphitic carbon nitride photocatalysts: the "holy grail" for photocatalytic hydrogen evolution reaction under visible light, Energy Environ. Sci, 2019, DOI: 10.1039/c9ee00717b.

8 K. Reilly, B. Fang, F. Taghipour and D. P. Wilkinson, Enhanced photocatalytic hydrogen production in a UVirradiated fluidized bed reactor, J. Catal., 2017, 353, 63-73.

9 R. Marschall, Photocatalysis: Semiconductor Composites: Strategies for Enhancing Charge Carrier Separation to Improve Photocatalytic Activity, Adv. Funct. Mater., 2014, 24, 2421-2440.

10 (a) J. Tang, J. R. Durrant and D. R. Klug, Mechanism of photocatalytic water splitting in $\mathrm{TiO}_{2}$. Reaction of water with photoholes, importance of charge carrier dynamics, and evidence for four-hole Chemistry, J. Am. Chem. Soc., 2008, 130, 13885-13891; (b) A. Kudo and Y. Miseki, Heterogeneous photocatalyst materials for water splitting, Chem. Soc. Rev., 2009, 38, 253-278; (c) M. Hoffmann, S. Martin, W. Choi and D. Bahnemann, Environmental applications of semiconductor photocatalysis, Chem. Rev., 1995, 95, 69-96.

11 W.-K. Jo and R. J. Tayade, New Generation Energy-Efficient Light Source for Photocatalysis: LEDs for Environmental Applications, Ind. Eng. Chem. Res., 2014, 53, 2073-2084.
12 B. Kundys, M. Viret, D. Colson and D. O. Kundys, Lightinduced size changes in $\mathrm{BiFeO}_{3}$ crystals, Nat. Mater., 2010, 9, 803-805.

13 (a) F. Gao, X. Chen, K. Yin, S. Dong, Z. F. Ren, F. Yuan, T. Yu, Z. G. Zou and J. M. Liu, Visible-light photocatalytic properties of weak magnetic $\mathrm{BiFeO}_{3}$ nanoparticles, $A d v$. Mater., 2007, 19, 2889-2892; (b) R. Guo, L. Fang, W. Dong, F. Zheng and $\mathrm{M}$. Shen, Magnetically Separable $\mathrm{BiFeO}_{3}$ nanoparticles with a $\gamma-\mathrm{Fe}_{2} \mathrm{O}_{3}$ parasitic phase: controlled fabrication and enhanced visible-light photocatalytic activity, J. Mater. Chem., 2011, 21, 18645-18652; (c) C. Reitz, C. Suchomski, C. Weidmann and T. Brezesinski, Block copolymer-templated $\mathrm{BiFeO}_{3}$ nanoarchitectures composed of phase-pure crystallites intermingled with a continuous mesoporosity: effective visible-light photocatalysts, Nano Res., 2011, 4, 414-424.

14 (a) J. An, L. Zhu, N. Wang, Z. Song, Z. Yang, D. Du and H. Tang, Photo-Fenton like degradation of tetrabromobisphenol A with graphene single bond $\mathrm{BiFeO}_{3}$ composite as a catalyst, Chem. Eng. J., 2013, 219, 225-237; (b) Z. Li, Y. Shen, C. Yang, Y. Lei, Y. Guan, Y. Lin, D. Liu and C. W. Nan, Significant enhancement in the visible light photocatalytic properties of $\mathrm{BiFeO}_{3}$-graphene nanohybrids, J. Mater. Chem. A, 2013, 1, 823-829.

15 O. P. Bajpai, S. Mandal, R. Ananthakrishnan, P. Mandal, D. Khastgir and S. Chattopadhyay, Structural features, magnetic properties and photocatalytic activity of bismuth ferrite nanoparticles grafted on graphene nanosheets, New J. Chem., 2018, 42, 10712-10723.

16 (a) X. Qi, J. Dho, R. Tomov, M. G. Blamire and J. L. M. Driscoll, Greatly reduced leakage current and conduction mechanism in aliovalent-ion-doped $\mathrm{BiFeO}_{3}$, Appl. Phys. Lett., 2005, 86, 062903-062905; (b) C. C. Chen, Z. X. Liu, G. Wang and Y. L. Yan, Pr and Gd co-doped bismuth ferrite thin films with enhanced multiferroic properties, Bull. Mater. Sci., 2014, 37, 1725-1729.

17 F. Sabeen, S. A. Irfan, M. Z. Iqbal and S. Rizwan, The high photocatalytic activity and reduced band gap energy of La and Mn-doped/graphene nanoplatelet (GNP) nanohybrids, RSC Adv., 2017, 7, 35928-35937.

18 H. Z. Qiang, M. Li, Y. Yu, J. Liu, L. Pei, J. Wang, X. Liu, B. Yu and $\mathrm{X}$. Zhao, Effects of $\mathrm{Nd}$ and high valence $\mathrm{Mn}$ co-doping on the electrical and magnetic properties of $\mathrm{BiFeO}_{3}$ ceramic, Solid State Commun., 2010, 150, 1088-1091; A. Kudo and Y. Miseki, Heterogeneous photocatalyst materials for water splitting, Chem. Soc. Rev., 2009, 38, 253-278; M. Hoffmann, S. Martin, W. Choi and D. Bahnemann, Environmental applications of semiconductor photocatalysis, Chem. Rev., 1995, 95, 69-96.

19 S. A. Ansari and M. H. Cho, Highly visible light responsive, narrow band gap $\mathrm{TiO}_{2}$ nanoparticles modified by elemental red phosphorus for photocatalysis and photoelectrochemical applications, Sci. Rep., 2016, 6, 25405-25414.

20 S. Fatima, S. I. Ali, D. Younas, A. Islam, D. Akinwande and S. Rizwan, Graphene nanohybrids for enhanced catalytic 
activity and large surface area, MRS Commun., 2018, 194, 110.

21 E. Vasilaki, I. Georgaki, D. Vernardou, M. Vamvakaki and N. Katsarakis, Ag-loaded $\mathrm{TiO}_{2} /$ reduced graphene oxide nanocomposites for enhanced visible-light photocatalytic activity, Appl. Surf. Sci., 2015, 353, 865-872.

$22 \mathrm{~W}$. Zhong, W. Tu, S. Feng and A. Xu, Photocatalytic $\mathrm{H}_{2}$ evolution on CdS nanoparticles by loading FeSe nanorods as co-catalyst under visible light irradiation, J. Alloys Compd., 2019, 772, 669-674.

23 L. Pai, Q. Chen, Y. Lin, G. Chang and Y. He, Effects of crystalline structure and interface band alignment on the photocatalytic property of bismuth ferrite/(N-doped) graphene composites, J. Alloys Compd., 2016, 672, 497-504.

24 F. Zhang, G. Randhorn, L. Chanman, A. Linda, S. T. J. Irvine and K. J. R. Peter, Assessment of oxidative visible light and UV active photocatalysts by hydroxyl radical quantification, J. Photochem. Photobiol., A, 2017, 334, 13-19.

25 J. F. Dai, T. Xian, L. J. Di and H. Yang, Preparation of $\mathrm{BiFeO}_{3}$ Graphene Nanocomposites and Their Enhanced Photocatalytic Activities, J. Nanomater., 2013, 2013, 642897-642901.

26 S. Irfan, L. L. Li, A. S. Saleemi and C.-W. Nan, Enhanced photocatalytic activity of $\mathrm{La}^{3+}$ and $\mathrm{Se}^{4+}$ co-doped bismuth ferrite nanostructures, J. Mater. Chem. A, 2017, 5, 1114311151.

27 F. Gao, X. Chen, K. Yin, S. Dong, Z. F. Ren, F. Yuan, T. Yu, Z. G. Zou and J. M. Liu, Visible-light photocatalytic properties of weak magnetic $\mathrm{BiFeO}_{3}$ nanoparticles, $A d v$. Mater., 2007, 19, 2889-2892.

28 R. Guo, L. Fang, W. Dong, F. Zheng and M. Shen, Magnetically separable $\mathrm{BiFeO}_{3}$ nanoparticles with a $\gamma$ $\mathrm{Fe}_{2} \mathrm{O}_{3}$ parasitic phase: controlled fabrication and enhanced visible-light photocatalytic activity, J. Mater. Chem., 2011, 21, 18645-18652; C. Reitz, C. Suchomski, C. Weidmann and T. Brezesinski, Block copolymer-templated $\mathrm{BiFeO}_{3}$ nanoarchitectures composed of phase-pure crystallites intermingled with a continuous mesoporosity: effective visible-light photocatalysts, Nano Res., 2011, 4, 414-424.

29 C. P. Kumar and M. Kar, Effect of rhombohedral to orthorhombic transition on magnetic and dielectric properties of La and Ti co-substituted $\mathrm{BiFeO}_{3}$, Smart Mater. Struct., 2015, 24, 045028-045031.

30 Y. Y. Gurkan, E. Kasapbasi and Z. Cinar, Enhanced solar photocatalytic activity of $\mathrm{TiO}_{2}$ by selenium (IV) ion-doping: Characterization and DFT modeling of the surface, Chem. Eng. J., 2013, 214, 34-44.

31 P. Tang, D. Kuang, S. Yang and Y. Zhang, The structural, optical and enhanced magnetic properties of $\mathrm{Bi}_{1-x} \mathrm{Gd}_{x} \mathrm{Fe}_{1-y} \mathrm{Mn}_{\mathrm{y}} \mathrm{O}_{3}$ nanoparticles synthesized by sol-gel, J. Alloys Compd., 2015, 622, 194-199.

32 R. Palai, R. S. Katiyar, H. Schmid, P. Tissot, S. J. Clark, J. Robertson, S. A. T. Redfern, G. Catalan and J. F. Scott, $\beta$ phase and $\gamma-\beta$ metal-insulator transition in multiferroic $\mathrm{BiFeO}_{3}$, Phys. Rev. B: Condens. Matter Mater. Phys., 2008, 77, 014110-014121.
33 G. Singh and L. N. K. Verma, Magnetoelectric coupling in multiferroic Tb-doped $\mathrm{BiFeO}_{3}$ nanoparticles, Mater. Lett., 2013, 111, 55-58.

34 Y. B. Yao and C. L. Mak, Optical, ferroelectric and magnetic properties of multiferroelectric $\mathrm{BiFeO}_{3}-\left(\mathrm{K}_{0.5} \mathrm{Na}_{0.5}\right)_{0.4}$ $\left(\mathrm{Sr}_{0.6} \mathrm{Ba}_{0.4}\right)_{0.8} \mathrm{Nb}_{2} \mathrm{O}_{6}$ thin films, J. Alloys Compd., 2014, 586, 448-455.

35 A. Ganguly, S. Sharma, P. Papakonstantinou and J. Hamilton, Probing the Thermal Deoxygenation of Graphene Oxide Using High-Resolution In Situ X-ray-Based Spectroscopies, J. Phys. Chem. C, 2011, 115, 17009-17019.

36 S. Chauhan, M. Kumar, S. Chhoker, S. C. Katyal and M. Singh, Substitution driven structural and magnetic transformation in Ca-doped $\mathrm{BiFeO}_{3}$ nanoparticles, $R S C$ Adv., 2016, 6, 43080-43090.

37 S. C. Das, S. Katiyal and T. Shripathi, Impedance spectroscopy of Bi-rich $\mathrm{BiFeO}_{3}$ : twin thermal-activations, $J$. Appl. Phys., 2018, 124, 174101-174105.

38 M. F. Sunding, K. Hadidi, S. Diplas, O. M. Løvvik, T. E. Norby and A. E. Gunnæs, XPS characterisation of in situ treated lanthanum oxide and hydroxide using tailored charge referencing and peak fitting procedures, Fuel and Energy Abstracts, 2011, 184, 399-409.

39 S. Chen, B. Pan, L. Zeng, S. Luo, X. X. Wang and W. Su, $\mathrm{La}_{2} \mathrm{Sn}_{2} \mathrm{O}_{7}$ enhanced photocatalytic $\mathrm{CO}_{2}$ reduction with $\mathrm{H}_{2} \mathrm{O}$ by deposition of Au co-catalyst, RSC Adv., 2017, 7, 1418614191.

40 M. Wanwan, R. Hu, J. Yang, Y. Du, J. Li and H. Wang, Influence of lanthanum-doping on photocatalytic properties of $\mathrm{BiFeO}_{3}$ for phenol degradation, Chin. J. Catal., 2016, 37, 1283-1292.

41 R. Das, T. Sarkar and K. Mandal, Multiferroic properties of $\mathrm{Ba}^{2+}$ and $\mathrm{Gd}^{3+}$ co-doped bismuth ferrite: magnetic, ferroelectric and impedance spectroscopic analysis, J. Phys D Appl. Phys., 2012, 45, 455002-455005.

42 M. Shenasa, S. Sainkar and D. Lichtman, XPS study of some selected selenium compounds, J. Electron Spectrosc. Relat. Phenom., 1986, 40, 329-337.

43 Y. Li, Y. Zhang, W. Le, J. Yu, C. Lu and L. Xia, Photo-tocurrent response of $\mathrm{Bi}_{2} \mathrm{Fe}_{4} \mathrm{O}_{9}$ nanocrystals synthesized through a chemical co-precipitation process, New J. Chem., 2012, 36, 1297-1300.

44 X. Y. Guan, Z. W. Qiao and D. Z. Yan, Investigation on structural and functional properties of $\mathrm{Nd}$ doped $\mathrm{BiFeO}_{3}$ particles prepared by hydrothermal method, J. Mater. Sci.: Mater. Electron., 2015, 26, 6807-6810.

$45 \mathrm{M}$. Sero and J. Bisquert, Fermi level of surface states in $\mathrm{TiO}_{2}$ nanoparticles, Nano Lett., 2003, 3, 945-948.

46 P. Vanga, R. Mangalaraja and M. Ashok, J. Mater. Sci.: Mater. Electron., 2016, 27, 5699-5706.

47 X. S. Xu, T. V. Brinzari, S. Lee, Y. H. Chu, L. W. Martin, A. Kumar and S. W. Cheong, Optical properties and magnetochromism in multiferroic $\mathrm{BiFeO}_{3}$, Phys. Rev. B: Condens. Matter Mater. Phys., 2009, 79, 134425-134433.

48 W. Zhong, Z. Lin, S. Feng, D. Wang, S. Shen, Q. Zhang, L. Gu, Z. Wand and B. Feng, Improved Oxygen Evolution Activity of 
$\mathrm{IrO}_{2}$ by in situ Engineering of Ultra-Small Ir Sphere Shell Utilizing Pulse Laser, Nanoscale, 2019, 11, 4407-4413.

49 Y. Liu, S. Shen, J. Zhang, W. Zhong and X. Huang, $\mathrm{Cu}_{2}-\mathrm{xSe} /$ CdS composite photocatalyst with enhanced visible light photocatalysis activity, Appl. Surf. Sci., 2019, 478, 762-769.

50 W. Zhong, S. Shen, S. Feng, Z. Lin, Z. Wanga and B. Fang, Facile fabrication of alveolate $\mathrm{Cu}_{2}-\mathrm{xSe}$ microsheets as a new visible-light photocatalyst for discoloration of Rhodamine B, CrystEngComm, 2018, 20, 7851-7856.

51 Z. Zhang, H. Liu, Y. Lin, Y. Wei, Ce-W. Nan and X. Deng, Influence of La Doping on Magnetic and Optical Properties of Bismuth Ferrite Nanofibers, Nanomaterials, 2012, 238605, 1-5.

52 N. Zhang, D. Chen, F. Niu, S. Wang, L. Qin and Y. Huang, Enhanced visible light photocatalytic activity of Gd-doped $\mathrm{BiFeO}_{3}$ nanoparticles and mechanism insight, Sci. Rep., 2016, 6, 26467-26476.

53 F. Mushtaq, X. Z. Chen, M. Hoop, H. Torlakcik, E. Pellicer, J. Sort, C. Gattinoni, B. J. Nelson and S. Pane, Piezoelectrically Enhanced Photocatalysis with $\mathrm{BiFeO}_{3}$ Nanostructures for Efficient Water Remediation, iScience, 2018, 4, 236-246.

54 Y. Huo, Y. Jin and Y. Zhang, Citric acid assisted solvothermal synthesis of $\mathrm{BiFeO} 3$ microspheres with high visible-light photocatalytic activity, J. Mol. Catal. A: Chem., 2010, 331, 15-20.

55 P. Kubelka and F. Munk, Ein Beitrag zur Optik der Farbanstriche (contribution to the optic of paint), Z. Phys, 1931, 12, 593-601.
56 J. P. Xu, R. J. Zhang, Z. H. Chen, Z. Y. Wang, F. Zhang, X. Yu and L. Y. Chen, Optical properties of epitaxial $\mathrm{BiFeO}_{3}$ thin film grown on $\mathrm{SrRuO}_{3}$-buffered $\mathrm{SrTiO}_{3}$ substrate, Nanoscale Res. Lett., 2014, 9, 188-194.

57 S. Irfan, S. Rizwan, Y. Shen, R. Tomovska, S. Zulfiqar, M. I. Sarwar and C. W. Nan, Mesoporous template-free gyroid-like nanostructure based on La and Mn co-doped bismuth ferrites with improved photocatalytic activity, $R S C$ Adv., 2016, 115, 114183-114189.

58 M. Sakar, B. Subramanian, I. Bhaumik, P. K. Gupta and S. N. Jaisankar, Nanostructured $\mathrm{Bi}_{1-x} \mathrm{Gd}_{x} \mathrm{FeO}_{3}-$ multiferroic photocatalyst on its sunlight driven photocatalytic activity, RSC Adv., 2014, 32, 16871-16878.

59 S. Irfan, S. Rizwan, Y. Shen, L. Li, S. Butt and C. W. Nan, The gadolinium $\left(\mathrm{Gd}^{3+}\right)$ and Tin $\left(\mathrm{Sn}^{4+}\right)$ co-doped $\mathrm{BiFeO}_{3}$ Nanoparticles as new solar light active photocatalyst, Sci. Rep., 2017, 7, 42493-42505.

60 W. Choi, A. Termina and M. R. Hoffman, The role of metal ion dopants in quantum-sized $\mathrm{TiO}_{2}$ : correlation between photoreactivity and charge carrier recombination dynamics, J. Phys. Chem., 1994, 98, 13669-13679.

61 C. Yongjuan, H. Ge, L. Wei, Z. Li, R. Yuan, P. Liu and X. Fu, Reduction degree of reduced graphene oxide (RGO) dependence of photocatalytic hydrogen evolution performance over RGO/ZnIn $\mathrm{S}_{4}$ nanocomposites, Catal. Sci. Technol., 2013, 7, 1712-1717.

62 A. Iwase, Y. H. Ng, Y. Ishiguro, A. Kudo and R. Amal, Reduced graphene oxide as a solid-state electron mediator in Z-scheme photocatalytic water splitting under visible light, J. Am. Chem. Soc., 2011, 133, 11054-11057. 